\title{
The suitability of exhaled breath compounds to identify children with exercise induced bronchoconstriction is modulated by the use of inhaled corticosteroids
}

Mattienne van der Kamp, Jean Driessen, Marc Van Der Schee, Boony Thio

European Respiratory Journal 2019 54: PA5432; DOI: 10.1183/13993003.congress-2019.PA5432

\section{Abstract}

Introduction: Accurate and timely diagnosis of exercise induced bronchoconstriction (EIB) is important for disease management, especially in children. Exhaled Volatile Organic Compounds (VOCs) can reflect underlying disease activity in the airways potentially enabling non-invasive diagnosis.

Objectives: In this cross-sectional study we investigated whether exhaled VOCs can discriminate between children with and without EIB.

Methods: Exhaled breath was collected in 46 children with a clinical suspicion of EIB using the Breath Biopsy platform (Owlstone Medical). EIB was assessed at OCON sports-medical clinic by a standardised exercise challenge with pulmonary function testing prior to and after exercise. EIB was defined as a post-exercise drop in FEV1 $>13 \%$ (on ICS) or a $>20 \%$ FEV1 reduction (ICS naïve). VOCs were extracted from the chromatogram and matched to library-IDs. Significantly discriminating VOCs were input into a Canonical Discriminant Analysis to subsequently generate a ROC-Curve with AUC value.

Results: VOCs did not differentiate between EIB ( $n=21$, age $8.7 \pm 2.2 \mathrm{yrs}$ ) and controls $(n=25$, age 8.4 \pm 3.1 years), AUC $0.42 \pm 0.31$. Similar results were found in post-hoc analysis when limiting the analysis to subjects taking ICS ( $n=23$, AUC $0.65 \pm 0.12$ ). However, in ICS naïve subjects a significant difference was found ( $\mathrm{n}=23$, AUC $0.86 \pm 0.08$ ), this was driven by 5 VOCs.

Conclusions: Analysis of exhaled VOCs only discriminates patients with and without EIB if they are steroid naïve. Speculatively, this could be related to ICS related suppression of inflammation although results require confirmation in a larger study.

\section{Children - Asthma - diagnosis - Allergy}

\section{Footnotes}

Cite this article as: European Respiratory Journal 2019; 54: Suppl. 63, PA5432.

This is an ERS International Congress abstract. No full-text version is available. Further material to accompany this abstract may be available at www.ers-education.org (ERS member access only).

- Copyright (C)the authors 2019 